\title{
The usefulness of fluorodeoxyglucose-positron emission tomography as a preoperative diagnostic tool for thymic epithelial tumors
}

\author{
Tsutomu Tatematsu ${ }^{1,2}$, Katsuhiro Okuda ${ }^{\wedge}$, Yushi Saito ${ }^{1}$, Risa Oda ${ }^{2}$, Tadashi Sakane ${ }^{2}$, Keisuke Yokota ${ }^{2}$, \\ Katsuhiko Endo ${ }^{2}$, Ryoichi Nakanishi ${ }^{2}$ \\ ${ }^{1}$ Department of General Thoracic Surgery, Toyota Memorial Hospital, Toyota, Japan; ${ }^{2}$ Department of Oncology, Immunology and Surgery, Nagoya \\ City University Graduate School of Medical Sciences, Nagoya, Japan \\ Contributions: (I) Conception and design: T Tatematsu, K Okuda; (II) Administrative support: R Nakanishi; (III) Provision of study materials or \\ patients: T Tatematsu, Y Saito, K Yokota, K Endo; (IV) Collection and assembly of data: T Tatematsu, Y Saito, R Oda, T Sakane; (V) Data analysis \\ and interpretation: T Tatematsu, K Okuda; (VI) Manuscript writing: All authors; (VII) Final approval of manuscript: All authors. \\ Correspondence to: Katsuhiro Okuda, MD, PhD. Department of Oncology, Immunology and Surgery, Nagoya City University Graduate School of \\ Medical Sciences, 1-Kawasumi, Mizuho-cho, Mizuho-ku, Nagoya 467-8601, Japan. Email: kokuda@med.nagoya-cu.ac.jp.
}

\begin{abstract}
Background To investigate the usefulness of ${ }^{18} \mathrm{~F}$-fluorodeoxy glucose-positron emission tomography $\left({ }^{18} \mathrm{~F}\right.$-FDG PET) for the preoperative imaging diagnosis of malignant grade in thymic epithelial tumors (TETs) and the correlation between the maximum standardized uptake value (SUVmax) and tumor size in TETs.

Methods: We retrospectively investigated 51 patients with TETs performed ${ }^{18}$ F-FDG PET. The SUVmax was compared between thymic carcinomas and thymomas. We also evaluated the difference in the SUVmax limited to small TETs. In addition, the correlation between the SUVmax and the tumor size was evaluated.

Results: The mean SUVmax of thymic carcinomas $(n=12)$ and thymomas $(n=39)$ was $5.71 \pm 2.6$ and 3.08 \pm 1.4 , respectively. The SUVmax of thymic carcinomas was significantly higher than that of thymomas $(\mathrm{P}<0.001)$. The mean SUVmax of these small thymic carcinomas $(n=3)$ and thymomas $(n=13)$ was $2.97 \pm 0.24$ and $1.79 \pm 0.47$, respectively. The SUVmax of the small thymic carcinomas was significantly higher than that of the thymomas $(\mathrm{P}=0.001)$. We found a positive correlation between the SUVmax and the maximum tumor size of TETs (correlation coefficient: 0.632, $\mathrm{P}<0.001$ ).
\end{abstract}

Conclusions: ${ }^{18}$ F-FDG PET might be useful for evaluating the preoperative malignancy of TETs. Of note, the maximum tumor size should be considered when performing assessments by ${ }^{18} \mathrm{~F}-\mathrm{FDG}$ PET.

Keywords: ${ }^{18} \mathrm{~F}$-fluorodeoxy glucose-positron emission tomography $\left({ }^{18} \mathrm{~F}\right.$-FDG PET); thymic epithelial tumors (TETs); thymic carcinoma; preoperative diagnosis

Submitted Sep 15, 2020. Accepted for publication Nov 17, 2020.

doi: 10.21037 /gs-20-718

View this article at: http://dx.doi.org/10.21037/gs-20-718

\section{Introduction}

The chances of detecting thymic epithelial tumors (TETs) are increasing with the spread of computed tomography (CT) screening for lung cancer $(1,2)$. However, distinguishing between thymic carcinomas with thymomas, especially small tumors, by preoperative diagnostic imaging is very difficult. For this reason, it is difficult to select the patients who should be treated with a neoadjuvant treatment and we cannot select the adequate chemotherapy regimens. Limited surgery, such as partial and subtotal thymectomy,

^ ORCID: 0000-0003-0099-2348. 
can be performed to manage small thymoma (3-5). In cases of small thymic carcinoma, however, expanded resection, including lymph node dissection, is needed because thymic carcinoma is more likely to invade locally and spread via the regional lymphatic system than thymoma with nonmyasthenia gravis (MG) (6). A more reliable diagnostic tool is thus required to determine the appropriate procedure.

The usefulness of ${ }^{18} \mathrm{~F}$-fluorodeoxy glucose-positron emission tomography $\left({ }^{18} \mathrm{~F}-\mathrm{FDG} \mathrm{PET}\right)$ for predicting the malignant grade in TETs has been reported (7-12). These previous reports suggested that the FDG uptake of thymic carcinomas was higher than that of thymomas. However, there have been few studies focusing on the tumor size of TETs.

In the present study, we investigated the usefulness of ${ }^{18}$ F-FDG PET for the preoperative imaging diagnosis of TETs as a novel diagnostic tool. Furthermore, the correlation between the maximum standardized uptake value (SUVmax) and tumor size was evaluated. We present the following article in accordance with the STARD reporting checklist (available at http://dx.doi.org/10.21037/ gs-20-718).

\section{Methods}

\section{Patients}

We retrospectively investigated 51 patients with TETs diagnosed from 2005 to 2018 at Toyota Memorial Hospital. The clinical background characteristics, postoperative pathological diagnosis, Masaoka stage and ${ }^{18} \mathrm{~F}$-FDG uptake in each case were investigated. The study was conducted in accordance with the Declaration of Helsinki (as revised in 2013). The study protocol was approved by the Institutional Review Board of Toyota Memorial Hospital and Nagoya City University Graduate School (No. 60190126) and individual consent for this retrospective analysis was waived. This study was registered on the UMIN Clinical Trial database (ID: 000040365).

\section{PET/computed tomography (CT)}

PET/CT was performed for all patients. These patients fasted for at least 5 hours before injection of 18F-FDG. $\mathrm{PET} / \mathrm{CT}$ image acquisition started at $60 \mathrm{~min}$ after intravenous injection of $4.3 \mathrm{MBq} / \mathrm{kg}$ of ${ }^{18} \mathrm{~F}$-FDG. Image acquisition was performed with biograph S16 (Siemens Medical Systems, USA). The PET/CT scan was performed from the groin to the forehead of patients, scanning by 150 sec per a bed and 6-8 bed per a patient. Acquired data were reconstructed by ordered subset expectation maximization (OSEM).

\section{Statistical analyses}

The FDG uptake (SUVmax) of thymomas and thymic carcinomas was compared using the $t$-test. The correlation between the SUVmax and the tumor size of those lesions was evaluated by Pearson's product moment correlation coefficient. All statistical analyses were performed using the EZR software program (13). Significance was defined as a probability value of less than 0.05 .

\section{Results}

The characteristics of the 51 patients are shown in Table 1 . There were 34 males and 17 females, and the median age was 56 years old (range, 44-67 years old). The 12 (23.5\%) cases diagnosed as thymic carcinoma included 11 squamous cell carcinomas and 1 neuroendocrine carcinoma. There were $39(76.5 \%)$ thymomas. Thymomas with MG were three cases. The World Health Organization (WHO) histological subtype was type $\mathrm{A}$ in 3 , type $\mathrm{AB}$ in 7 , type $\mathrm{B} 1$ in 7 , type $\mathrm{B} 1 / \mathrm{B} 2$ in 1 , type $\mathrm{B} 2$ in 15 , type $\mathrm{B} 3$ in 4 and micronodular thymoma with lymphoid stroma in 2 . The Masaoka stage was stage I in 26 , stage II in 14 , stage III in 7 , stage IVa in 3 and stage IVb in 1 . The median maximum tumor size was $3.4 \mathrm{~cm}$ (range, 0.9 to $10 \mathrm{~cm}$ ). The operative approach was median sternotomy in 27 , videoassisted thoracic surgery in 23 , thoracotomy via lateral incision in 1 . The operative procedure of 39 thymomas was extended thymectomy in 4 , total thymectomy in 23 , subtotal thymectomy in 3 and partial thymectomy in 9 . The operative procedure of 12 thymic carcinoma was total thymectomy in 8 , subtotal thymectomy in 1 and partial thymectomy in 3. All thymomas with MG were performed extended thymectomy. Only one case was diagnosed with thymic carcinoma preoperatively.

The SUVmax was compared between the 12 thymic carcinomas and 39 thymomas using ${ }^{18} \mathrm{~F}-\mathrm{FDG}$ PET. The mean SUVmax of thymic carcinomas and thymomas was $5.71 \pm 2.6$ and $3.08 \pm 1.4$, respectively. The SUVmax of thymic carcinomas was significantly higher than that of thymomas $(\mathrm{P}<0.001)$ (Figure 1). Although the SUVmax was compared between the low-risk thymomas (type $\mathrm{A}, \mathrm{AB}$, and B1) and high-risk thymomas (type B2 and B3), there was no 
Table 1 The clinical and pathological characteristics of patients with thymic epithelial tumors

\begin{tabular}{|c|c|}
\hline Variables & Total $(n=51)$ \\
\hline \multicolumn{2}{|l|}{ Sex } \\
\hline Male/female & $34 / 17$ \\
\hline \multicolumn{2}{|l|}{ Age (years) } \\
\hline Median [range] & 56 [44-67] \\
\hline \multicolumn{2}{|l|}{ Pathological diagnosis } \\
\hline Thymic carcinoma & 12 \\
\hline Squamous cell carcinoma & 11 \\
\hline Neuroendocrine carcinoma & 1 \\
\hline Thymoma & 39 \\
\hline Type A & 3 \\
\hline$A B$ & 7 \\
\hline B1 & 7 \\
\hline B2 & 15 \\
\hline B1/B2 & 1 \\
\hline B3 & 4 \\
\hline Micronodular thymoma with lymphoid stroma & 2 \\
\hline \multicolumn{2}{|l|}{ Pathological Masaoka stage } \\
\hline I & 26 \\
\hline II & 14 \\
\hline III & 7 \\
\hline IVa & 3 \\
\hline $\mathrm{IVb}$ & 1 \\
\hline \multicolumn{2}{|l|}{ Maximum tumor size $(\mathrm{cm})$} \\
\hline Median [range] & $3.4[0.9-10]$ \\
\hline
\end{tabular}

statistical difference between these two groups of thymoma $(\mathrm{P}=0.37)$.

Among the analyzed TETs in this study, we identified 3 thymic carcinomas and 13 thymomas with a maximum tumor size of $\leq 2 \mathrm{~cm}$. The mean SUVmax of these small thymic carcinomas and thymomas was $2.97 \pm 0.24$ and $1.79 \pm 0.47$, respectively. The SUVmax of the small thymic carcinomas was significantly higher than that of the thymomas ( $\mathrm{P}=0.001)$ (Figure 2). The SUVmax of small TETs was lower than that of the larger TETs (Figure 3).

Regarding the correlation between the SUVmax and TET sizes, a positive correlation was recognized between

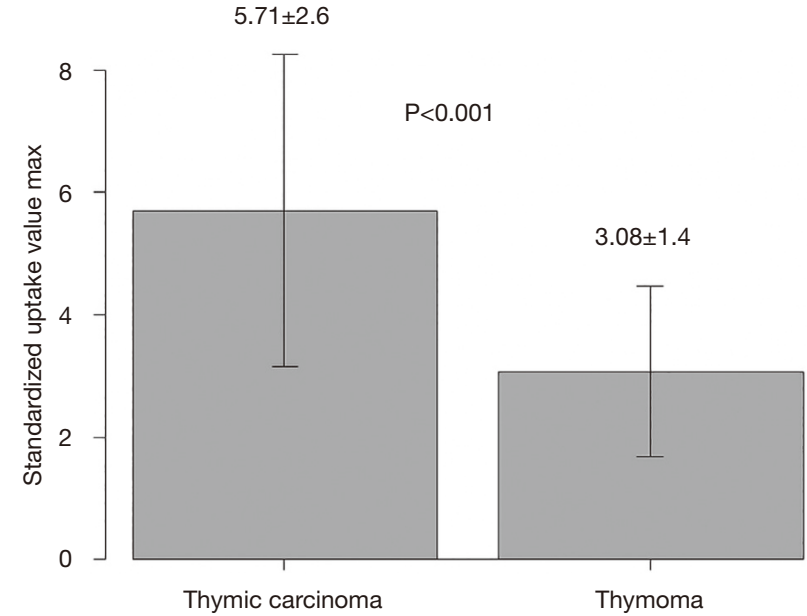

Figure 1 The maximum standardized uptake value of thymic carcinoma and thymoma. Data are expressed as mean \pm SD. A statistically significant difference was defined as $\mathrm{P}<0.05$. SD, standard deviation.

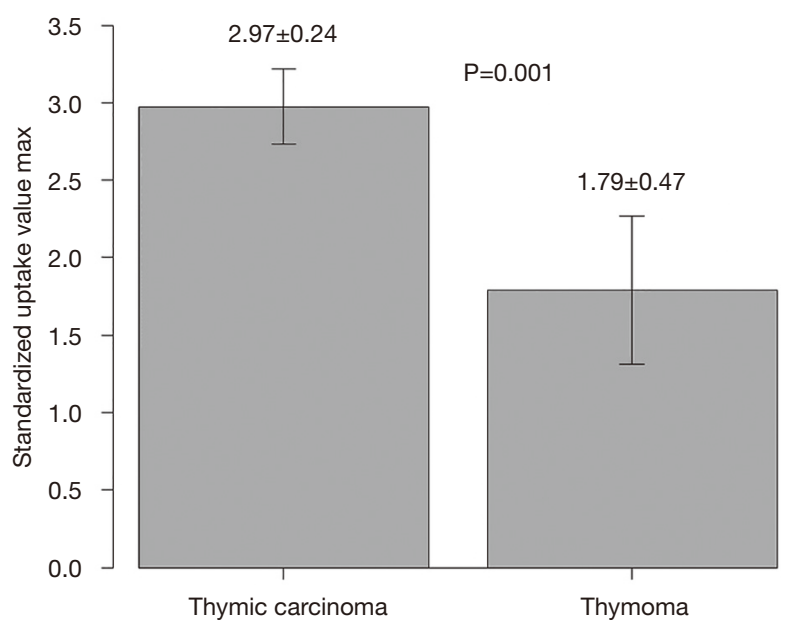

Figure 2 The maximum standardized uptake value small thymic epithelial tumors (maximum tumor size $\leq 2 \mathrm{~cm}$ ). Data are expressed as mean \pm SD. A statistically significant difference was defined as $\mathrm{P}<0.05$. SD, standard deviation.

the two variables (correlation coefficient: $0.632, \mathrm{P}<0.001$ ) (Figure 4). Similar results were obtained in the group divided into thymic carcinomas with thymomas (Figure 5).

A receiver operating curve (ROC) analysis was conducted for the differential diagnosis of thymic carcinoma and thymoma using the SUVmax (Figure 6). When the SUVmax of 5.23 was used as a cut-off value, the sensitivity and 


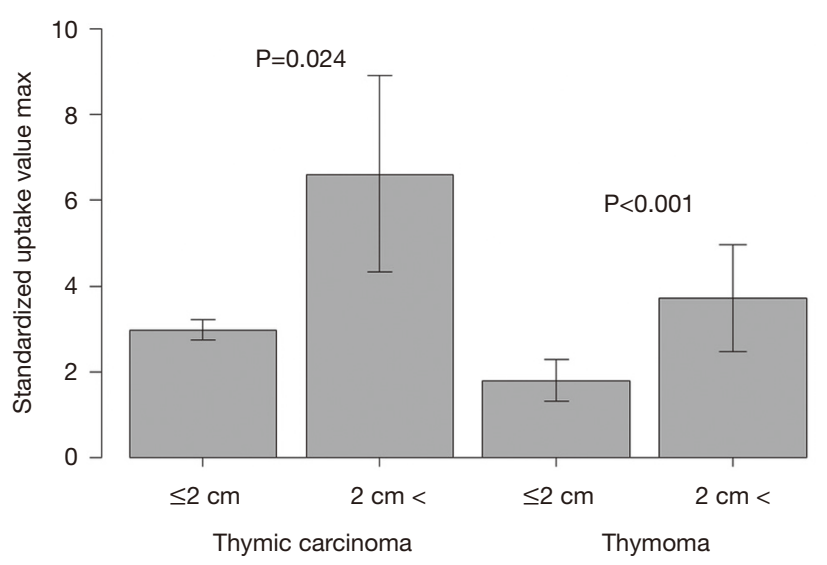

Figure 3 Differences in the maximum standardized uptake value between small and large thymic epithelial tumors. A statistically significant difference was defined as $\mathrm{P}<0.05$.

Correlation coefficient: 0.632 $\mathrm{P}<0.001$

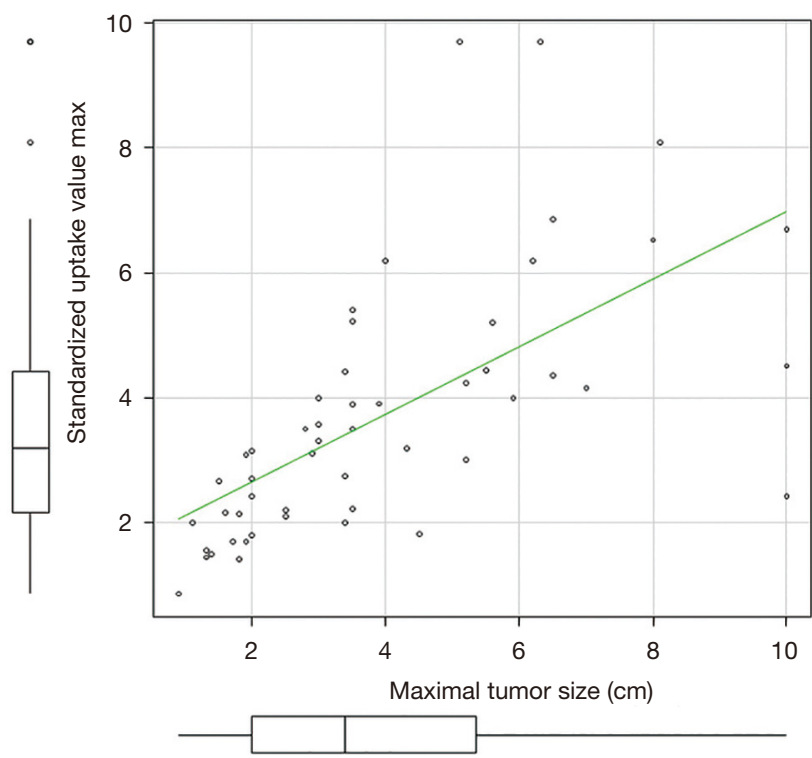

Figure 4 The correlation between the maximum standardized uptake value and the maximum tumor size in thymic epithelial tumors. A statistically significant difference was defined as $\mathrm{P}<0.05$.

specificity were $92.3 \%$ and $58.3 \%$, respectively, and the area under the curve (AUC) was 0.811 [95\% confidence interval (CI), 0.672-0.95] (Figure 6A). Regarding the cases with a maximum tumor size of $\leq 2 \mathrm{~cm}$, when the SUVmax of 2.70 was used as a cut-off value, the sensitivity and specificity were $100 \%$ and $100 \%$, respectively, and the AUC was 1

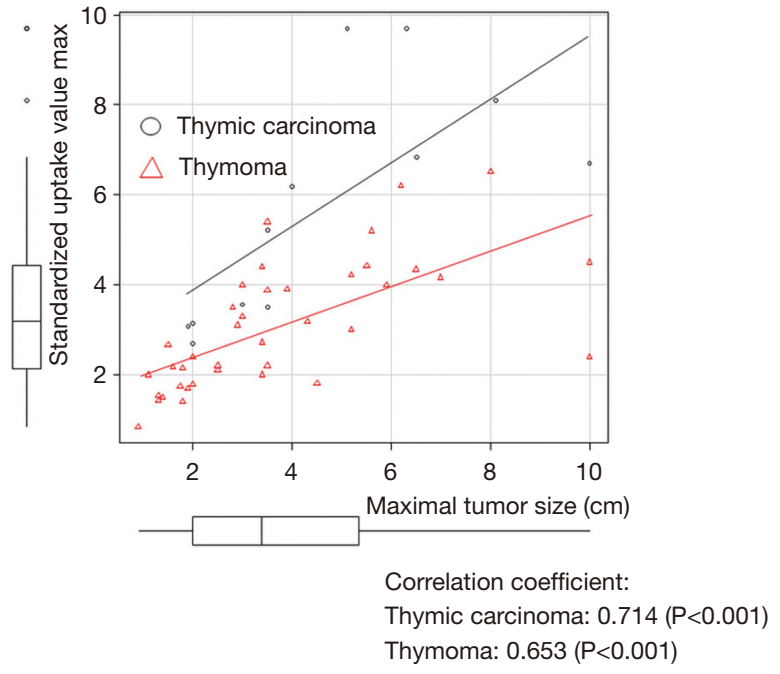

Figure 5 The correlation between the maximum standardized uptake value and the maximum tumor size in thymic carcinoma and thymoma. A statistically significant difference was defined as $\mathrm{P}<0.05$.

(95\% CI, 1-1) (Figure 6B). Furthermore, for cases with maximum tumor size of $\leq 3 \mathrm{~cm}$ (4 thymic carcinoma and 19 thymoma), when the SUVmax of 2.70 was used as a cut-off value, the sensitivity and specificity were $78.9 \%$ and $100 \%$, respectively, and the AUC was 0.842 (95\% CI, 0.679-1) (Figure 6C).

\section{Discussion}

Regarding ${ }^{18} \mathrm{~F}$-FDG PET in the preoperative imaging diagnosis of TETs, it was suggested that the SUVmax of thymic carcinoma was higher than that of thymoma, but there was no significant difference in these values between high- and low-risk thymoma in this study. Even for small TETs, the SUVmax was significantly different between thymic carcinomas and thymomas, although the values were not very high. The correlation between the SUVmax and tumor size for TETs was found to be positive. We also evaluated the cut-off value of SUVmax for differentiating thymoma from thymic carcinoma by an ROC analysis and found that the cut-off value for tumors of all sizes was 5.23, while that for small tumors ( $\leq 2$ and $\leq 3 \mathrm{~cm}$ ) was 2.70 . The accuracy seemed to differ between tumor $\leq 2$ and $\leq 3 \mathrm{~cm}$ in size (AUC 1, 95\% CI: 1-1 vs. AUC 0.842, 95\% CI: 0.6791). Therefore, when using the SUVmax to differentiate thymomas from thymic carcinomas, it may be necessary to set the appropriate cut-off value according to the maximum 
A

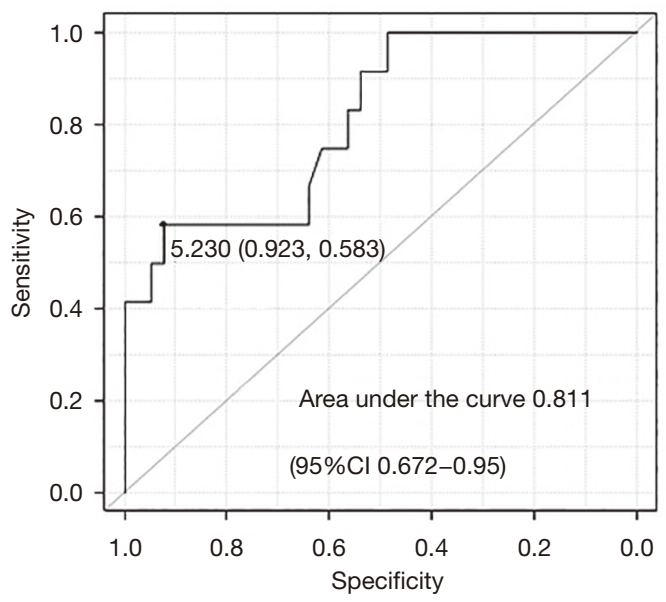

C

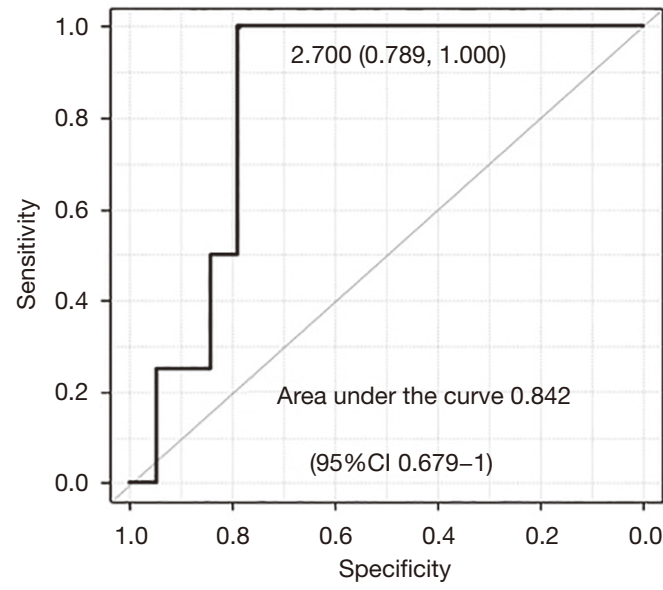

B

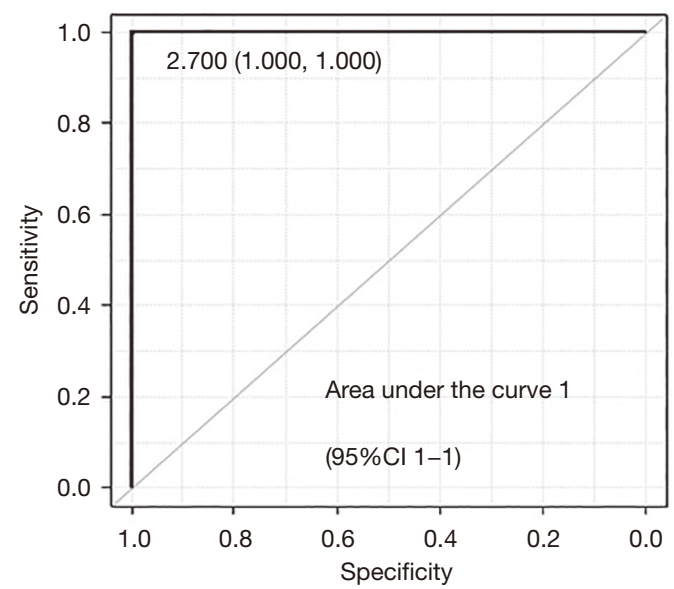

Figure 6 A receiver operating curve for the differential diagnosis of thymic carcinoma and thymoma using the maximum standardized uptake value. (A) All cases; (B) the cases with a maximum tumor size of $\leq 2 \mathrm{~cm}$; (C) the cases with a maximum tumor size of $\leq 3 \mathrm{~cm}$. CI, confidence interval.

tumor size.

These results were different from previous reports (9-12). Park et al. reported that there was no correlation between tumor size and the SUVmax for 61 patients with TETs (54 thymomas and 7 thymic carcinomas) (11). Furthermore, Nakagawa et al. explored the cut-off value of SUVmax for differentiating thymoma from thymic carcinoma in 112 patients with TETs (92 thymoma and 20 thymic carcinoma) (12). In their report, the SUVmax of 4.58 was used as a cut-off value with a sensitivity of $80 \%$ and a specificity of $78.3 \%$. Regarding TETs of $\leq 3 \mathrm{~cm}$, the SUVmax of 4.57 was used as a cut-off value with a sensitivity of $75.0 \%$ and a specificity of $93.8 \%$. There therefore seems to be no correlation between the SUVmax and tumor size.

Why these results from previous studies differed from our own might be because our study had a higher proportion of small tumors than the other studies. Indeed, in Park's report, the mean tumor size was $6.1 \pm 3.4 \mathrm{~cm}$. In Nakagawa's report, the median tumor size was $5.0 \mathrm{~cm}$ (range: $1.3-13.0 \mathrm{~cm})$, and $20(18 \%)$ out of 112 cases were small TETs $(\leq 3 \mathrm{~cm})$. In our study, the maximum tumor size was $3.4 \mathrm{~cm}$ (range: $0.9-10.0 \mathrm{~cm}$ ), and $16(31.3 \%$ ) of the 51 cases were $\leq 2 \mathrm{~cm}$, including, 3 (5.9\%) cases of thymic carcinomas. Lococo et al. analyzed the data of 47 patients with TETs in multicenter and reported that 
SUVmax and SUVmax/tumor size were predictive factors in distinguishing thymomas from thymic carcinomas (14). Tomita et al. also reported that both SUVmax and SUVmax/ tumor size were useful for differentiating low-risk thymoma (A, AB, B1) from high-risk-thymoma (B2, B3) and thymoma from thymic carcinoma in 73 patients with TETs (15). Moreover, both parameters significantly correlated with Masaoka stage. Korst et al. reported the only prospective study using the International Thymic Malignancy Interest Group (ITMIG) database (16). In this report, the value of SUVmax that maximizes sensitivity and specificity in the prediction of $\mathrm{WHO}$ histologic type (A, AB, B1, B2 vs. B3, carcinoma) was 5.55 (sensitivity, 81\% and specificity, 66\%). There was a very weak correlation between maximum tumor size and SUVmax.

Given the present findings, regarding small TETs, even if the SUVmax is relatively low, the possibility of thymic carcinoma should be considered. In clinical practice, when thymic carcinoma is suspected in cases of small TETs, it might be necessary to diagnose pathologically whether it is thymic carcinoma or thymoma during surgery. If the lesion is found to be thymic carcinoma, it will then be necessary to determine the suitable extent of resection.

However, our study had some limitations. First, there were only a small number of patients with TETs. A largescale, prospective study of TETs should be conducted to evaluate the usefulness of ${ }^{18} \mathrm{~F}-\mathrm{FDG}$ PET for the preoperative diagnostic imaging of TETs. Second, ${ }^{18} \mathrm{~F}-\mathrm{FDG}$ PET might not have actually been performed for TETs, as our study was retrospective. In addition, we investigated only patients with resected TETs, and un-resected TETs were not included, so there might have been some selection bias. Third, especially small tumor-size may alter PET image quantitation because of the partial volume effect, which most strongly affects the smallest structures due to the poor spatial resolution of PET.

In conclusion, ${ }^{18}$ F-FDG PET might be useful for the preoperative evaluation of TETs. However, the tumor size of TETs should be considered when conducting assessments using ${ }^{18}$ F-FDG PET.

\section{Acknowledgments}

Funding: None.

\section{Footnote}

Reporting Checklist: The authors have completed the STARD reporting checklist. Available at http://dx.doi.org/10.21037/ gs-20-718

Data Sharing Statement: Available at http://dx.doi. org/10.21037/gs-20-718

Conflicts of Interest: All authors have completed the ICMJE uniform disclosure form (available at http://dx.doi. org/10.21037/gs-20-718). The authors have no conflicts of interest to declare.

Ethical Statement: The authors are accountable for all aspects of the work in ensuring that questions related to the accuracy or integrity of any part of the work are appropriately investigated and resolved. The study was conducted in accordance with the Declaration of Helsinki (as revised in 2013). The study protocol was approved by the Institutional Review Board of Toyota Memorial Hospital and Nagoya City University Graduate School (No. 60190126) and individual consent for this retrospective analysis was waived). This study was registered on the UMIN Clinical Trial database (ID: 000040365).

Open Access Statement: This is an Open Access article distributed in accordance with the Creative Commons Attribution-NonCommercial-NoDerivs 4.0 International License (CC BY-NC-ND 4.0), which permits the noncommercial replication and distribution of the article with the strict proviso that no changes or edits are made and the original work is properly cited (including links to both the formal publication through the relevant DOI and the license). See: https://creativecommons.org/licenses/by-nc-nd/4.0/.

\section{References}

1. The National Lung Screening Trial Research Team, Aberle DR, Adams AM, et al. Reduced lung-cancer mortality with low-dose computed tomographic screening N Engl J Med 2011;365:395-409.

2. Xiang D, Zhang B, Doll D, et al. Lung cancer screening: from imaging to biomarker. Biomark Res 2013;1:4.

3. Yano M, Fujii Y, Yoshida J, et al. A phase II study of partical and subtotal thymectomy for thymoma (JART02). World J surg 2017;41:2033-8.

4. Odaka M, Akiba T, Yabe M, et al. Unilateral thoracoscopic subtotal thymectomy for the treatment of stage I and II thymoma. Eur J Cardiothorac Surg 2010;37:824-6.

5. Sakamaki Y, Kido T, Yasukawa M. Alternative choices of 
total and partial thymectomy in video-assisted resection of noninvasive thymomas. Surg Endosc 2008;22:1272-7.

6. Okuma Y, Hosomi Y, Watanabe K, et al. Clinicopathological analysis of thymic malignancies with a consistent retrospective database in a single institution: from Tokyo Metropolitan Cancer Center. BMC Cancer 2014;14:349-57.

7. Liu RS, Yeh SH, Huang MH, et al. Use of fluorine-18 fluorodeoxyglucose positron emission tomography in the detection of thymoma: a preliminary report. Eur J Nucl Med 1995;22:1402-7.

8. Kubota K, Yamada S, Kondo T, et al. PET imaging of primary mediastinal tumors. Br J Cancer 1996;73:882-6.

9. Sung YM, Lee KS, Kim BT, et al. 18F-FDG PET/CT of thymic epithelial tumors: usefulness for distinguishing and staging tumor subgroups. J Nucl Med 2006;47:1628-34.

10. Kim JY, Kim HO, Kim JS, et al. 18F-FDG PET/CT is useful for pretreatment assessment of the histopathologic type of thymic epithelial tumors. Nucl Med Mol Imaging 2010;44:177-84.

11. Park SY, Cho A, Bae MK, et al. Value of 18F-FDG PET/ CT for predicting the world health organization malignant

Cite this article as: Tatematsu T, Okuda K, Saito Y, Oda R, Sakane T, Yokota K, Endo K, Nakanishi R. The usefulness of fluorodeoxyglucose-positron emission tomography as a preoperative diagnostic tool for thymic epithelial tumors. Gland Surg 2021;10(2):690-696. doi: 10.21037/gs-20-718 grade of thymic epithelial tumors focused in volumedependent parameters. Clin Nucl Med 2016;41:15-20.

12. Nakagawa K, Takahashi S, Endo M, et al. Can 18F-FDG PET predict the grade of malignancy in thymic epithelial tumors? An evaluation of only resected tumors. Cancer Manag Res 2017;9:761-8.

13. Kanda Y. Investigation of the freely available easy-touse software 'EZR' for medical statistics. Bone Marrow Transplant 2013;48:452-8.

14. Lococo F, Cesario A, Okami J, et al. Role of combined 18F-FDG-PET/CT for predicting the WHO malignancy grade of thymic epithelial tumors: a multicenter analysis. Lung Cancer 2013;82:245-51.

15. Tomita M, Ayabe T, Tsuchiya K, et al. Fluorodeoxyglucose Positron Emission Tomography Can Provide Useful Information for Differentiating Thymic Epithelial Tumors. Thorac Cardiovasc Surg 2018;66:345-9.

16. Korst RJ, Fernando S, Catlin AC, et al. Positron Emission Tomography in Thymic Tumors: Analysis Using a Prospective Research Database. Ann Thorac Surg 2017;104:1815-20. 